\title{
Microscopic Findings Sponsor Defined Identifier
}

National Cancer Institute

\section{Source}

National Cancer Institute. Microscopic Findings Sponsor Defined Identifier. NCI

Thesaurus. Code C117576.

One or more sponsor defined characters used to identify, name, or characterize the microscopic findings. 